\title{
Reconnaître, traiter et signaler les cas de thrombocytopénie immunitaire thrombotique induite par un vaccin
}

\author{
Michelle Sholzberg MDCM MSc, Donald M. Arnold MD MSc, Andreas Laupacis MD MSc
}

Citation : CMAJ 2021 June 14;193:E913-5. doi : 10.1503/cmaj.210882-f; diffusion hâtive le 14 mai 2021

Voir la version anglaise de l'article ici : www.cmaj.ca/lookup/doi/10.1503/cmaj.210882; voir l'article connexe en français au www.cmaj. ca/lookup/doi/10.1503/cmaj.210795-f, et en anglais au www.cmaj.ca/lookup/doi/10.1503/cmaj.210795

D

ans un rapport connexe, Jones et ses collaborateurs décrivent le cas d'un homme de 63 ans qui a présenté des thromboses des artères poplitées et pulmonaires ${ }^{1}$. Ces thromboses étaient probablement imputables à une thrombocytopénie immunitaire thrombotique induite par le vaccin (TTIV), une rare complication du vaccin ChAdOx1 nCoV-19 (Oxford-AstraZeneca), qu'il avait reçu 20 jours auparavant ${ }^{1}$. Nous présentons certaines leçons importantes que les médecins peuvent tirer de ce rapport de cas.

Les thromboses secondaires à la TTIV sont souvent atypiques et peuvent être fatales. Des cas ont été signalés dans diverses veines et artères, y compris dans les veines cérébrales ${ }^{2,3}$. Des TTIV et des thromboses associées ont été observées jusqu'à 30 jours après l'administration d'une dose de vaccin à vecteur adénoviral contre le SRAS-CoV-2. La pathophysiologie de la TTIV est encore obscure, mais un élément propre aux composants adénoviraux du vaccin semble stimuler la réaction immunitaire responsable de la TTIV4,5.

Le vaccin à vecteur adénoviral qui a été largement utilisé au Canada est le ChAdOx1 nCoV-19 (Oxford-AstraZeneca). L'Alberta et l'Ontario en ont suspendu l'utilisation le 11 mai 2021, et d'autres provinces leur ont emboîté le pas. Pour l'instant, la meilleure estimation de l'incidence mondiale de la TTIV consécutive à une première dose du vaccin ChAdOx1 nCoV-19 se situe entre 1 pour 26000 et 1 pour 127000 cas $^{6}$. Comme les médecins sont de plus en plus conscients de la TTIV et qu'ils la diagnostiquent, la traitent et la signalent plus souvent, l'incidence pourrait augmenter. La TTIV semble moins courante après la seconde dose de vaccin à vecteur adénoviral, même si des cas ont été rapportés ${ }^{6}$. Le risque de TTIV semble moindre avec le vaccin de Johnson and Johnson ${ }^{6}$ et, à notre connaissance, aucun cas n'a été décrit après l'administration des vaccins à ARN messager couramment utilisés ${ }^{6}$.

La TTIV peut être un syndrome clinique agressif, et selon les rapports publiés à ce jour, 20\%-50\% des personnes affectées

\section{POINTS CLÉS}

- Des cas de thrombocytopénie immunitaire thrombotique induite par le vaccin (TTIV) et de thromboses associées ont été signalés jusqu'à 30 jours après l'administration d'une dose de vaccin à vecteur adénoviral contre le SRAS-CoV-2, comme le ChAdOx1 nCoV-19 (Oxford-AstraZeneca).

- Les thromboses secondaires à la TTIV sont souvent atypiques et peuvent toucher le réseau veineux et artériel; selon les rapports enregistrés à ce jour, $20 \%-50 \%$ des personnes victimes d'une TTIV en meurent.

- Même si la TTIV est rare, les médecins doivent maintenir un fort degré de suspicion à l'endroit de ce syndrome clinique chez les patients qui manifestent des symptômes évocateurs de thrombose et disent avoir reçu un vaccin à vecteur adénoviral.

- Pour le dépistage de la TTIV, les médecins devraient utiliser le test ELISA (enzyme-linked immunosorbent assay) appliqué à la thrombocytopénie induite par l'héparine; le diagnostic est confirmé par un dosage fonctionnel de la sérotonine plaquettaire modifié avec l'ajout du facteur plaquettaire 4, mais le traitement doit débuter avant même la confirmation du diagnostic, idéalement sous la supervision d'un hématologue.

- Il est important de déclarer tous les cas de TTIV aux instances régionales de la santé publique appropriées pour mieux comprendre la nature du syndrome et l'innocuité des vaccins.

en meurent ${ }^{2-5}$. Étant donné les graves conséquences cliniques de la TTIV, les médecins doivent maintenir un fort degré de suspicion à son égard chez les patients qui manifestent des symptômes évoquant ceux de la thrombose d'un vaisseau, quel qu'il soit, dans les 30 jours suivant l'administration d'un vaccin à vecteur adénoviral contre le SRAS-CoV-2, malgré la faible incidence de cette affection. Un diagnostic précoce est plus susceptible d'être posé si les médecins s'informent systématiquement du type et du moment de la vaccination contre le SRAS-CoV-2 
lors de l'anamnèse. Les médecins devraient consulter des experts sans tarder et envisager un transfert vers un centre doté d'une unité de soins intensifs et d'un service d'immunohématologie spécialisée.

Les importants signes et symptômes de la thrombose associée à la TTIV comprennent ce qui suit : céphalées intenses et persistantes; vision trouble ou double; faiblesse ou altération de la sensibilité d'un côté du corps; douleur à la poitrine, à l'abdomen, à la jambe ou au dos; enflure des jambes; et essoufflement. Il faut demander une formule sanguine complète chez tous les patients soupçonnés de présenter une TTIV ${ }^{6-9}$. La présence d'une thrombocytopénie ou d'une thrombose chez un patient qui a reçu un vaccin à vecteur viral dans les 30 jours précédents augmente nettement la probabilité d'une TTIV. Les taux de $D$-dimères et de fibrinogène peuvent aider les médecins à évaluer les signes de coagulopathie de consommation ${ }^{9}$. En effet, un début de TTIV a été signalé chez des patients qui avaient seulement un taux de plaquettes bas, un taux de D-dimères élevé ou un taux de fibrinogène bas, même sans thrombose ${ }^{10}$.

Pour le dépistage de la TTIV, on utilise le même test ELISA (enzyme-linked immunosorbent assay) que pour la thrombocytopénie induite par l'héparine (TIH), qui se révèle positif chez la plupart des patients atteints de TTIV ${ }^{5,9}$. Les auteurs du rapport de cas connexe ont raison de mentionner que les autres immunodosages (p. ex., au latex) utilisés pour repérer les cas de TIH sont généralement négatifs chez les patients atteints de TTIV. Les médecins doivent se renseigner sur le test utilisé dans leur hôpital parce que de nombreux établissements canadiens n'utilisent pas le test ELISA ${ }^{6,9}$. Le diagnostic de TTIV peut être confirmé au moyen d'un dosage fonctionnel de la sérotonine plaquettaire modifié par l'ajout du facteur plaquettaire 4 (PF4), effectué au laboratoire d'immunologie plaquettaire de I'Université McMaster (Hamilton, Ont.). Par contre, face à un diagnostic présumé de TTIV ${ }^{6}$, les médecins doivent rapidement instaurer le traitement approprié, préférablement sous la supervision d'un hématologue, sans attendre les résultats des tests de confirmation.

Selon une hypothèse actuelle, la TTIV se manifeste parce qu'un anticorps anti-PF4 déclenche une activation plaquettaire qui entraîne une thrombose et une thrombocytopénie de consommation, imitant le mécanisme observé dans la TIH, mais sans exposition à l'héparine. Comme la TTIV est récente, les recommandations thérapeutiques sont les mêmes que pour le traitement de la TIH et s'inspirent des leçons tirées de l'expérience acquise au chevet des patients atteints de TTIV, peu nombreux, mais dont le nombre augmente. Le traitement doit viser la thrombose et le dérèglement immunitaire du patient. En théorie, il est possible que l'héparine exacerbe la TTIV parce que les anticorps en cause dans la TTIV risquent de réagir aux complexes PF4-héparine circulants ${ }^{1-8,10-12}$. Par conséquent, la thrombose doit plutôt être traitée au moyen d'anticoagulants non hépariniques, comme l'argatroban, le fondaparinux ou un anticoagulant oral direct. La prise en charge est compliquée si la thrombose liée à la TTIV s'accompagne d'hémorragies, particulièrement chez les patients présentant une thrombose des sinus veineux cérébraux ou un $A V C^{4,8,10}$.
On a utilisé l'immunoglobuline intraveineuse pour le traitement de la TTIV sur la base de l'expérience acquise auprès des patients atteints de $\mathrm{TIH}$ et de thrombose graves ${ }^{2-5,7,8,12}$. On croit que l'immunoglobuline intraveineuse neutralise et inhibe l'action pathogène des anticorps impliqués dans la TTIV, et ce faisant, fait augmenter la numération plaquettaire. Il faut prélever les échantillons sanguins pour les tests de la TIH (ELISA et dosages de la sérotonine) avant d'administrer l'immunoglobuline intraveineuse ${ }^{9}$. Chez les patients dont l'état se détériore malgré le traitement, on pourrait devoir recourir à l'aphérèse pour éliminer les anticorps circulants impliqués dans la TTIV2-5,7,8,12. On peut aussi envisager l'administration de corticostéroïdes pour atténuer la réaction immunitaire aberrante $e^{4,7,8,12}$. Il faut éviter les transfusions de plaquettes en raison du risque d'aggraver le syndrome clinique en fournissant plus d'antigène (c.-à-d., PF4) aux anticorps pathogènes circulants; elles ne devraient être envisagées que sur les conseils d'un hématologue $e^{2,3,5,7,8,12}$.

Étant donné l'incertitude relative à l'incidence, à la pathophysiologie, aux caractéristiques cliniques et à la prise en charge à court et à long terme de la TTIV, il est important de reconnaître et de signaler tous les cas de TTIV aux instances régionales de la santé publique au Canada (https://www.canada .ca/fr/sante-publique/services/immunisation/federale-provinciale -territoriale-renseignements-personnes-contact-questions-liees -effets-secondaires-suivant-immunisation.html). Un meilleur signalement nous aidera à mieux comprendre la nature de la TTIV, à la traiter optimalement et à caractériser l'innocuité des vaccins.

\section{Références}

1. Jones M, Boisvert A, Landry J, et al. Limb ischemia and pulmonary artery thrombosis after the ChAdOx1 nCoV-19 (Oxford-AstraZeneca) vaccine: a case of vaccine-induced immune thrombotic thrombocytopenia. CMAJ 2021 May 14 [cyberpublication avant impression]. doi: 10.1503/cmaj.210795.

2. Greinacher A, Thiele T, Warkentin TE, et al. Thrombotic thrombocytopenia after ChAdOx1 nCov-19 vaccination. N Engl J Med 2021 Apr 9;NEJMoa2104840. doi: 10.1056/NEJMoa2104840 [cyberpublication avant impression]. Accessible ici : https://www.nejm.org/doi/10.1056/NEJMoa2104840 (consulté le 10 mai 2021).

3. Schultz NH, Sørvoll IH, Michelsen AE, et al. Thrombosis and thrombocytopenia after ChAdOx1 nCoV-19 vaccination. N Engl J Med 2021 Apr. 9. doi: 10.1056/ NEJMoa2104882 [cyberpublication avant impression]. Accessible ici : https:// www.nejm.org/doi/10.1056/NEJMoa2104882 (consulté le 10 mai 2021).

4. Cines DB, Bussel JB. SARS-CoV-2 vaccine-induced immune thrombotic thrombocytopenia. N Engl J Med 2021 Apr 16;NEJMe2106315. doi: 10.1056/NEJMe2106315 [cyberpublication avant impression].

5. Scully M, Singh D, Lown R, et al. Pathologic antibodies to platelet factor 4 after ChAdOx1 nCoV-19 vaccination. N Engl J Med 2021 Apr. 16. doi: 10.1056/ NEJMoa2105385 [cyberpublication avant impression]. Accessible ici : https:// www.nejm.org/doi/10.1056/NEJMoa2105385 (consulté le 10 mai 2021).

6. Pai M, Chan B, Stall NM, et al.; Drugs \& Biologics Clinical Practice Guidelines Working Group and the Ontario COVID-19 Science Advisory Table. Vaccine-induced immune thrombotic thrombocytopenia (VITT) following adenovirus vector COVID-19 vaccination. Ontario: Ontario COVID-19 Science Advisory Table; 2021 May 7. Accessible ici : https://covid19-sciencetable.ca/sciencebrief/vaccine -induced-immune-thrombotic-thrombocytopenia-vitt-following-adenovirus-vector -covid-19-vaccination/ (consulté le 9 mai 2021).

7. Guidance produced from the Expert Haematology Panel (EHP) focused on syndrome of thrombosis and thrombocytopenia occurring after coronavirus vaccination. London (UK): British Society for Haematology; 2021 May 20. Accessible ici : https://b-s-h.org.uk/about-us/news/guidance-produced-by-the-expert-haematology -panel-ehp-focussed-on-vaccine-induced-thrombosis-and-thrombocytopenia-vitt/ (consulté le 9 mai 2021). 
8. Oldenburg J, Klamroth R, Langer F, et al. Diagnosis and management of vaccinerelated thrombosis following AstraZeneca COVID-19 vaccination: guidance statement from the GTH. Hamostaseologie 2021 Apr 1. doi: 10.1055/a-1469-7481 [cyberpublication avant impression].

9. Nazy I, Sachs UJ, Arnold DM, et al. Recommendations for the clinical and laboratory diagnosis of vaccine-induced immune thrombotic thrombocytopenia (VITT) for SARS-CoV-2 infections: communication from the ISTH SSC Subcommittee on Platelet Immunology. J Thromb Haemostasis 2021 Apr. 22. doi: https://doi. org/10.1111/jth.15341 [cyberpublication avant impression]. Accessible ici : https:// onlinelibrary.wiley.com/doi/abs/10.1111/jth.15341 (consulté le 9 mai 2021).

10. Thaler J, Ay C, Gleixner KV, et al. Successful treatment of vaccine-induced prothrombotic immune thrombocytopenia (VIPIT J Thromb Haemostasis 2021 Apr. 20. doi: https://doi.org/10.1111/jth.15346 [cyberpublication avant impression]. Accessible ici : https://onlinelibrary.wiley.com/doi/abs/10.1111/jth.15346 (consulté le 9 mai 2021).

11. Coronavirus vaccine - weekly summary of Yellow Card reporting. London (UK): Medicines and Healthcare products Regulatory Agency - Department of Health and Social Care; 2021 May 6. Accessible ici : https://www.gov.uk/government/ publications/coronavirus-covid-19-vaccine-adverse-reactions/coronavirus-vaccine -summary-of-yellow-card-reporting (consulté le 9 mai 2021).

12. Bussel JB, Connors JM, Cines DB, et al. Thrombosis with thrombocytopenia syndrome. Hematology.org; updated 2021 Apr. 29. Accessible ici : https:// www.hematology.org:443/covid-19/vaccine-induced-immune-thrombotic -thrombocytopenia (consulté le 9 mai 2021).

\section{Intérêts concurrents : Aucun déclaré.}

Cet article a été sollicité et il n'a pas été révisé par des pairs.

Affiliations : Départements de médecine, de médecine de laboratoire et de pathobiologie (Sholzberg), Hôpital St. Michael's, Université de Toronto; Département de médecine (Arnold), Université McMaster, Toronto, Ont.; rédacteur adjoint principal (Laupacis), CMAJ, Ottawa, Ont.

Collaborateurs : Tous les auteurs ont contribué à la conception du travail, ont rédigé le manuscrit et en ont révisé de façon critique le contenu intellectuel important; ils ont donné leur approbation finale pour la version destinée à être publiée et assument l'entière responsabilité de tous les aspects du travail.

Propriété intellectuelle du contenu : Il s'agit d'un article en libre accès distribué conformément aux modalités de la licence Creative Commons Attribution (CC BY-NC-ND 4,0), qui permet l'utilisation, la diffusion et la reproduction dans tout médium à la condition que la publication originale soit adéquatement citée, que l'utilisation se fasse à des fins non commerciales (c.-à-d., recherche ou éducation) et qu'aucune modification ni adaptation n'y soit apportée. Voir : https://creativecommons.org/ licenses/by-nc-nd/4.0/deed.fr.

Remerciements : Les auteurs tiennent à souligner la contribution éditoriale de Grace Tang, Hôpital St. Michael's, Institut de gestion, d'évaluation et de politiques de santé, Université de Toronto.

Avis : Andreas Laupacis est rédacteur adjoint principal du CMAJ et n'a pas participé au processus ayant mené au choix de cet article.

Correspondance : Michelle Sholzberg, michelle.sholzberg@unityhealth.to 